\title{
The Association between Central Obesity and the Risk of Irritable Bowel Syndrome: A Case-control Study
}

\author{
Bilal Natiq Nuaman* \\ Department of Medicine, College of Medicine, Al-Iraqia University Baghdad, Iraq \\ *Corresponding author: Bilal_natiq@yahoo.com
}

\begin{abstract}
Background: Central obesity and Irritable Bowel Syndrome (IBS) are common medical conditions with similar etiologic mechanisms. There are limited studies to evaluate the association between obesity, especially central obesity; with IBS. Establishing such association is important in the management of IBS. Objective: To evaluate the association between central obesity and IBS in Iraqi patients using Waist-to-Height ratio (WtHR) as an index of central obesity. Patients and Methods: Central obesity defined by Waist-to-Height ratio $>0.5$. Inclusion and exclusion criteria for diagnosing IBS were used. A case-control study was performed at AL-Nuaman Teaching Hospital in the period from January 2015 to December 2015, Comparing Waist-to-Height ratio (WtHR) between 75 patients (with IBS) and 130 controls (without IBS) to assess the association between central obesity and IBS. Chi-square test with $95 \%$ confidence was used to evaluate association between central obesity and IBS, and Odds ratio (OR) for central obesity was determined. Results: There is significant association between central obesity and IBS, $\left.x^{2}(1)=36.2, \mathrm{P}<0.001\right)$. The strength of association using phi coefficient is medium positive $(\varphi=42, \mathrm{p}<0.001)$. Conclusion: Central obesity is significantly associated with IBS and may be regarded as a risk factor for IBS.
\end{abstract}

\begin{tabular}{|l|l|}
\hline OR & value \\
\hline Odds Ratio for Central Obesity (Central Obesity / No-Central Obesity) & 6.403 \\
\hline For cohort IBS = IBS & 2.972 \\
\hline For cohort IBS = No-IBS & .464 \\
\hline
\end{tabular}

Keywords: central obesity, IBS, Waist-to-Height ratio (WtHR), case-control study

Cite This Article: Bilal Natiq Nuaman, "The Association between Central Obesity and the Risk of Irritable Bowel Syndrome: A Case-control Study." American Journal of Medical Sciences and Medicine, vol. 5, no. x (2017): 21-23. doi: 10.12691/ajmsm-5-2-1.

\section{Introduction}

Irritable bowel syndrome (IBS) is a common chronic functional gastrointestinal disorder with a prevalence of up to $45 \%$ [1]. Diagnosis of IBS depends on patients' presentation of recurrent abdominal pain and altered bowel frequency with the exclusion of any organic disease. There are many mechanisms have been implicated in the pathogenesis of IBS including diet, microbiota, inflammation, and psychosocial functioning $[2,3,4]$.

Obesity is a common chronic endocrine disorder associated with many diseases including those related to GIT mainly GERD $[5,6,7]$, but there are limited studies to prove association with IBS. Pathogenesis of obesity is multifactorial and includes also diet, microbiota, inflammation, and psychological factors [8]. The dysmetabolic syndrome as a result of an inflammatory activity is greater in patients with visceral obesity even with normal Body Mass Index (BMI) [9].

Waist-to-Height ratio (WtHR) [termed also as Index of Central Obesity (ICO)], with a value of more than 0.5 [10], is strongly associated with visceral fattiness measured via DEXA [11], making it as the best index for central or abdominal obesity.

There are some researches to evaluate the association between visceral abdominal obesity and irritable bowel syndrome, but to my knowledge no study uses WtHR or ICO in defining central obesity. Moreover no survey was performed in Iraq to investigate the relationship between central obesity and having Irritable Bowel Syndrome. Therefore, the aim of this case-control study is to evaluate the association between Central Obesity and the risk of Irritable Bowel Syndrome.

\section{Methods}

\subsection{Study Design}

A Case-Control study was conducted in the outpatient department of internal medicine of AL-Nuaman Teaching Hospital in the period from January 2015 to December 2015. Any person with age more than 50 years was excluded from both case and control groups. 


\subsection{Inclusion Criteria}

There are many criteria for diagnosing IBS, but Rome III Diagnostic Criteria are useful in clinical practice and can be used to make a positive clinical diagnosis with high sensitivity and specificity $[12,13]$. These criteria include abdominal pain for more than 6 months, which becomes recurrent at least 3 days per month in the last 3 months, with 2 or more of the following features must fulfill: (1) Pain is relieved by defecation; (2) Pain associated with a change in frequency of stool; and (3) Pain associated with a change in form of stool.

\subsection{Exclusion Criteria}

There are many features which make diagnosis of organic rather than IBS.

These include: (1) Fever, Weight loss or Jaundice; (2) Change in the color of stool; (3) Signs of anemia or Thyroid disease; (4) Abdominal mass or Organomegaly; (5) Signs of intestinal obstruction

\subsection{Anthropometric Measurements}

Waist Circumference (WC) and Height measured for patients and control groups. Then Waist-to-height ratio (WHtR) was determined, dividing WC (in $\mathrm{cm}$ ) by height (in $\mathrm{cm}$ ). A WHtR cutoff point of 0.50 was used in determining central obesity. Central obesity defined as having WHtR $>0.5$.

\subsection{Statistical Analysis}

The data was entered and examined entirely utilizing PC software of the Statistical Package for the Social
Science (SPSS) version 20.0. Cross-tabulation analysis (Chi-square test) with $95 \%$ confidence was used to evaluate association between central obesity and IBS, and Odds ratio (OR) for central obesity was determined including 95\% confidence interval [CI]. A P-value of $<$ 0.05 was been considered to indicate the level of significance throughout the study. Strength of association was measured using Phi coefficient.

\section{Results}

\subsection{Study Participants}

A total of 120 patients were participated with 45 patients excluded from this study. There were 75 patients (with IBS) and 130 persons (without IBS) as a control, enrolled in this study.

\subsection{Prevalence of Central Obesity}

Prevalence of central obesity in IBS group was more than 3 times in non- IBS group (Table 1), and most of control group have no central obesity (Figure 1).

\subsection{Association between Central Obesity and IBS}

There is significant association between central obesity and IBS, $\left.x^{2}(1)=36.2, \mathrm{P}<0.001\right)$.Patients with central obesity have a risk to have IBS 6 times more than those without central obesity (Table 2). The strength of association using phi coefficient is medium positive $(\varphi=42$, $\mathrm{p}<0.001$ ).

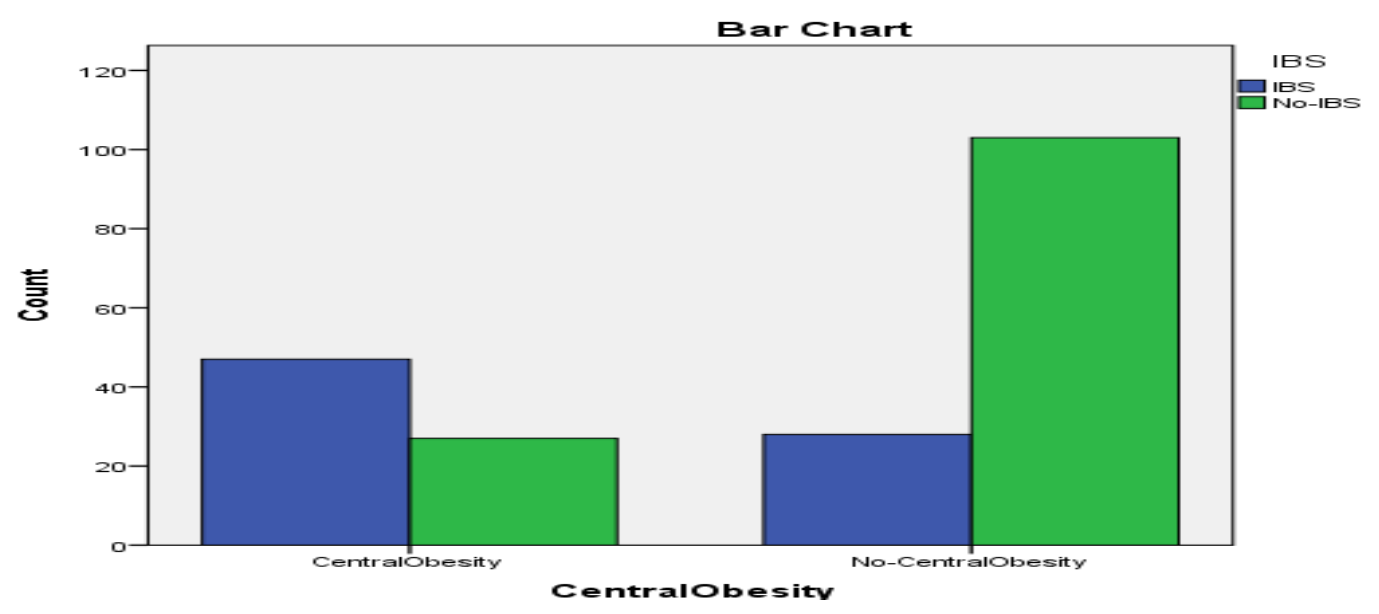

Figure 1. Distribution of central obesity in patients and controls

Table 1. Prevalence of central obesity among participants

\begin{tabular}{|c|c|c|c|c|c|}
\hline & & & \multicolumn{2}{|c|}{ IBS } & \multirow{2}{*}{ Total } \\
\hline & & & IBS & No-IBS & \\
\hline \multirow{6}{*}{ Central Obesity } & \multirow{3}{*}{ Central Obesity } & Count & 47 & 27 & 74 \\
\hline & & $\%$ within IBS & $62.7 \%$ & $20.8 \%$ & $36.1 \%$ \\
\hline & & $\%$ of Total & $22.9 \%$ & $13.2 \%$ & $36.1 \%$ \\
\hline & \multirow{3}{*}{ No-Central Obesity } & Count & 28 & 103 & 131 \\
\hline & & $\%$ within IBS & $37.3 \%$ & $79.2 \%$ & $63.9 \%$ \\
\hline & & $\%$ of Total & $13.7 \%$ & $50.2 \%$ & $63.9 \%$ \\
\hline
\end{tabular}


Table 2. Risk Estimation for IBS

\begin{tabular}{|c|c|c|c|}
\hline & \multirow{2}{*}{ Value } & \multicolumn{2}{|c|}{ 95\% Confidence Interval } \\
\hline & & Lower & Upper \\
\hline Odds Ratio for Central Obesity (Central Obesity / No-Central Obesity) & 6.403 & 3.406 & 12.038 \\
\hline For cohort IBS $=$ IBS & 2.972 & 2.050 & 4.307 \\
\hline For cohort IBS $=$ No-IBS & .464 & .339 & .635 \\
\hline
\end{tabular}

\section{Discussion}

My study indicates that prevalence of central obesity and IBS among all participants was $36.6 \%$ and $36.1 \%$ respectively. Prevalence of central obesity among IBS vs. non-IBS groups was $62.7 \%$ and $20.8 \%$ respectively. Central obesity was significantly associated with IBS (odds ratio (OR), 6.4; 95\% confidence interval [CI], 3.4 to 12.03).

Central obesity is associated with many medical conditions. There are several studies which had been studied the relationships between general obesity and symptoms of IBS, all these studies use BMI as an index of obesity, but very limited studies evaluated the association between central obesity and IBS. Indeed I found just a single case-control study to evaluate association between central obesity and IBS performed by Lee CG [14] who measure central obesity using abdomen computerized tomography (CT) and found that a higher visceral adipose tissue $(\mathrm{OR})=9.42)$ and waist circumference $(\mathrm{OR}=7.81)$ were associated with IBS.

My results are similar to those of most studies which show a positive association between IBS and general obesity [15,16,17]; and between IBS and central obesity [14]. Some studies show that central obesity using WC is more associated with IBS than general obesity $[18,19]$. However, there are some studies which did not show this association $[20,21,22]$. To my knowledge this is the first case-control study to evaluate the association between central obesity, using waist-to-height ratio (WHtR), and irritable bowel syndrome. Using waist-to-height ratio (WHtR) as an index of central obesity is a simple practical measure unlike the cost and risk of CT.

Several explanations for the association between IBS and central obesity, It has been suggested that obese persons have uninhibited satiety leading them to overeating of diets that are low in fiber, high in saturated fats and fermentable carbohydrates which may contribute to IBS symptoms [23]. On the other hand, the density of (ghrelin) endocrine cell type is increased in IBS. Ghrelin stimulates food intake leading to central obesity [24].

Alterations in the composition of the gut microbiota have been noticed in both obesity and IBS and may be a common association between the 2 disorders [25,26,27,28,29]. Low grade inflammation or immune activation has been postulated to be one of the mechanisms of IBS pathogenesis through effects on visceral sensitivity and gut epithelial function [30,31]. IBS is associated with higher serum tumor necrosis factor alpha (TNF- $\alpha$ ) levels and increased levels of the proinflammatory cytokines interleukins 6 (IL-6) [32]. Obesity associated with an increased number of adipocytes [33]. There are many evidences that these adipocytes secrete TNF- $\alpha$ and IL-6 in large quantities, which may contribute to insulin resistance and systemic inflammation of obesity $[34,35,36]$.
Further studies are needed to establish the firm association between central obesity and IBS, the proving of such association is important in management of IBS.

In addition to small sample size, one of limitations of this study is the sample not stratified by gender, and IBS patients not categorized into diarrhea predominant or constipation predominant.

\section{Conclusion}

The results of this research indicate a positive association between central obesity and IBS, and central obesity is a risk factor for IBS.

\section{Acknowledgements}

I would like to thank medical and nursing staff of outpatient department of al-Nuaman hospital.

\section{References}

[1] Lovell RM, Ford AC. Global prevalence of and risk factors for irritable bowel syndrome: A meta-analysis. Clin Gastroenterol Hepatol 2012; 10:712 21.

[2] Niec A, Frankum B, Talley N. Are adverse food reactions linked to irritable bowel syndrome? Am J Gastroenterol 1998; 93: 2184-90.

[3] Madden J, Hunter J. A review of the role of the gut microflora in irritable bowel syndrome and the effects of probiotics. Br J Nutr 2002; 88:S67-72.

[4] Ford AC, Talley NJ. Mucosal inflammation as a potential etiological factor in irritable bowel syndrome: A systematic review. J Gastroenterol 2011; 46: 421-31.

[5] Corley DA, Kubo A. Body mass index and gastroesophageal reflux disease: a systematic review and meta-analysis. Am J Gastroenterol. 2006; 101 (11): 2619-2628.

[6] Hampel H, Abraham N, El-Serag H. Meta-analysis: obesity and the risk for gastroesophageal reflux disease and its complications. Ann Intern Med. 2005; 143: 199.

[7] Locke GR, Talley NJ, Fett SL, et al. Risk factors associated with symptoms of gastroesophageal reflux. AmJ Med.1999; 106: 642-649.

[8] Gortmaker S, Must A, Perrin J, et al. Social and economic consequences of overweight in adolescence and young adulthood. N Engl J Med. 1993; 329: 1008-12.

[9] Ruderman N, Chisholm D, Pi-Sunyer X, et al. The metabolically obese, normal-weight individual revisited. Diabetes. 1998; 47: 699-713.

[10] Parikh RM, Joshi SR, Menon PS, Shah NS. Index of central obesity- A novel parameter. Medical Hypotheses. 2007; 68: 1272-1275.

[11] Ashwell M, Cole T, Dixon A. Ratio of waist circumference to height is strong predictor of intra-abdominal fat. BMJ. 1996; 313 : 559-560.

[12] Drossman D, Corrazziari E, Delvaux M, et al. Rome III: the functional Gastrointestinal Disorders. 3rd ed. McLean, VA: Degnon Associates; 2006.

[13] Tibble J, Sigthorsson G, Foster R, et al. Use of surrogate markers of inflammation and Rome criteria to distinguish organic from nonorganic intestinal disease.Gastroenterology 2002; 123: 450-60. 
[14] Sadik R, Björnsson E, Simrén M. The relationship between symptoms, body mass index, gastrointestinal transit and stool frequency in patients with irritable bowel syndrome. Eur J Gastroenterol Hepatol. 2010; 22(1): 102-108. 416 Gastroenterology \& Hepatology Volume 10, Issue 7 July 2014.

[15] Clements RH, Gonzalez QH, Foster A, et al. Gastrointestinal symptoms are more intense in morbidly obese patients and are improved with laparoscopic Rouxen-Y gastric bypass. Obes Surg. 2003; 13(4): 610614

[16] Roberson EN, Gould JC, Wald A. Urinary and fecal incontinence after bariatric surgery. Dig Dis Sci.2010; 55(9): 2606-2613.

[17] FysekidisM, Bouchoucha M, Bihan H, Reach G, Benamouzig R, Catheline JM. Prevalence and co-occurrence of upper and lower functional gastrointestinal symptoms in patients eligible for bariatric surgery. Obes Surg.2012; 22(3): 403-410.

[18] Simrén M, Månsson A, Langkilde AM, Svedlund J, Abrahamsson $\mathrm{H}$, Bengtsson U, Björnsson ES. Food-related gastrointestinal symptoms in the irritable bowel syndrome. Digestion. 2001; 63(2): 108-15.

[19] Lee SH, Kim KN, Kim KM, and Joo NS. Irritable Bowel Syndrome May Be Associated with Elevated Alanine Aminotransferase and Metabolic Syndrome. Yonsei Med J. 2016 Jan 1; 57(1): 146-152.

[20] Lee CG, Lee JK, Kang YS, Shin S, Kim JH, Lim YJ, et al. Visceral abdominal obesity is associated with an increased risk of irritable bowel syndrome. Am J Gastroenterol. 2015 Feb; 110(2): 310-9.

[21] Talley NJ, Howell S, Poulton R. Obesity and chronic gastrointestinal tract symptoms in young adults: a birth cohort study. Am J Gastroenterol. 2004; 99: 1807-14.

[22] Kubo M, Fujiwara Y, Shiba M, Kohata Y, Yamagami H, Tanigawa $\mathrm{T}$, et al. Differences between risk factors among irritable bowel syndrome subtypes in Japanese adults. Neurogastroenterol Motil. 2011 Mar; 23(3):249-54.

[23] Kang HW, Lee CG, Kim JH, Lim YJ, Lee JK, Koh MS, et al. Visceral abdominal obesity as a risk factor for irritable bowel syndrome: a case-control study. Gastroenterology. 2014; 146 (5 Suppl 1): S-178.
[24] Hosoda H, Kojima M, Kangawa K, Ghrelin and the regulation of food intake and energy balance. Mol Interv. 2002 Dec; 2(8): 494-503.

[25] Ley RE, Turnbaugh PJ, Klein S, Gordon JI. Microbial ecology: human gut microbes associated with obesity. Nature. 2006; 444 (7122): 1022-1023.

[26] Kassinen A, Krogius-Kurikka L, Mäkivuokko H, et al. The fecal microbiota of irritable bowel syndrome patients differs significantly from that of healthy subjects. Gastroenterology. 2007; 133(1): 24-33.

[27] Turnbaugh PJ, Bäckhed F, Fulton L, Gordon JI. Diet-induced obesity is linked to marked but reversible alterations in the mouse distal gut microbiome. Cell Host Microbe. 2008; 3(4): 213-223.

[28] Jeffery IB, Quigley EM, Öhman L, Simrén M, O’Toole PW. The microbiota link to irritable bowel syndrome: an emerging story. Gut Microbes. 2012; 3(6):572-576.

[29] Simrén M, Barbara G, Flint HJ, et al; Rome Foundation Committee. Intestinal microbiota in functional bowel disorders: a Rome foundation report. Gut. 2013; 62(1): 159-176.

[30] Mayer E, Collins S. Evolving pathophysiologic models functional gastrointestinal disorders. Gastroenterology2002; 122: 2032-48.

[31] Camilleri M. Peripheral mechanisms in irritable bowel syndrome. N Engl J Med 2012; 367: 1626-35.

[32] ScullyP, Mckernan DP, KeohaneJ, et al. Plasma cytokine profiles in females with irritable bowel syndrome and extra-intestinal comorbidity. Am J Gastroenterol 2010; 105: 2235.

[33] Hirsch J, Knittle JL. Cellularity of obese and non-obese human adipose tissue. Fed Proc. 1970; 29: 1516-1521.

[34] Peraldi P, Spiegelman B. TNF- $\alpha$ and insulin resistance: summary and future prospects. Mol Cell Biochem. 1998; 182: 169-171.

[35] Mohamed-Ali V, Pinkney JH, Coppack SW. Adipose tissue as an endocrine and paracrine organ. Int J Obes Relat Metab Disord. 1998; 22: 1145-1158.

[36] Bastard JP, Jardel C, Bruckert E, et al. Elevated levels of interleukin 6 are reduced in serum and subcutaneous adipose tissue of obese women after weight loss. J Clin Endocrinol Metab. 2000; 85: 3338-3342. 